\title{
Aplicación de las tecnologías de la información y la comunicación (TIC) en el proceso de exportación. orientado a las pequeñas y medianas empresas (PYMES) de Bucaramanga
}

\author{
Édgar Rodríguez Afanador ${ }^{1}$ \\ Luz Stella Garnica Sandoval ${ }^{2}$
}

\section{Resumen}

Las tecnologías de la información y la comunicación (TIC) han transformado la forma de administrar la información en las empresas, al volverse elementos dinamizadores en la creación de nuevos productos y servicios, y así generar valor agregado para sus clientes y competencias para el capital humano de las organizaciones. Su alcance abarca toda la cadena de valor de una empresa, lo que permite engranar aspectos externos y apropiar los contextos regionales sirviendo de apoyo para la toma de decisiones de una manera más rápida y acertada para la gerencia.

Por todo lo anterior, la presente investigación busca mostrar el avance en la aplicación de las TIC en las pequeñas y medianas empresas (PYMES) exportadoras de la ciudad de Bucaramanga, estableciendo la importancia que tiene para el desarrollo de sus actividades, el tipo de capacitación que tiene el personal, como también, las formas de comunicación que utilizan los microempresarios para mantenerse conectados con sus clientes, sus proveedores, sus asesores y las entidades gubernamentales. De igual forma, se quiere establecer el tipo de previsión financiera que realizan las PYMES para la compra y la reposición de las TIC, así como el tipo de infraestructura física y tecnológica que tienen actualmente para el intercambio de información entre los diferentes puestos de trabajo de las empresas.

Palabras clave: Tecnologías de la información y la comunicación (TIC), exportaciones, PYMES, previsión financiera, costos, infraestructura tecnológica.

\section{Abstract}

Information and Communication Technologies (ICT) have transformed the way of managing information in business, becoming the driving forces in creating new products and services, creating value for customers and competencies for the organization's human capital. Its scope covers the entire value chain of an enterprise, enabling gear externalities and appropriate to regional contexts, serving as support for decision-making, in a more rapid and accurate for management.

The research aims to show progress in the implementation of Information and Communication Technologies (ICT) in small and medium-sized exporters in the city of Bucaramanga, establishing the importance for the development of its activities, the type of training has the staff, as well as forms of communication they use to stay connected with customers, suppliers, consultants and government agencies. Likewise, set the type of financial projections made for the purchase and replacement of ICT by SMEs, the type physical and technological infrastructure, which currently have to exchange information among different jobs in the companies.

Key words: Information and communication technologies (ICT), exports, SMEs, financial forecasting, cost, technology infrastructure.

1 MBA Administration. Docente ocasional, UNAD Colombia. CEAD Bucaramanga. Grupo Visionarios, Bucaramanga (Colombia). Correo electrónico: edgara.rodriguez@unad.edu.co.

${ }^{2}$ MBA Administration. Docente ocasional, UNAD Colombia. CEAD Bucaramanga. Grupo Visionarios, Bucaramanga (Colombia). Correo electrónico: luz.garnica@unad.edu.co. 


\section{Introducción}

Una parte considerable de los grandes avances tecnológicos que ha tenido lugar desde fines del siglo XX está relacionada con el desarrollo de las tecnologías de la información y la comunicación $(\mathrm{TIC})^{3}$. Esta tendencia ha transformado la forma de hacer negocios y las formas de producir, y, por tanto, ha redefinido los mercados y transformado la vida de los clientes en todo el planeta.

Por todo lo anterior, el continuo avance de las TIC acrecentó la eficiencia de los procesos productivos por medio de la creación, la difusión, la recolección y el uso intensivo de información; además, propició el desarrollo de nuevos productos y servicios, y así facilitó la interconexión de los mercados y los capitales financieros en tiempo real ${ }^{4}$.

Como consecuencia de lo enunciado, las TIC se han transformado en la herramienta por excelencia para aumentar la productividad, sector donde se convierten en el eslabón para incidir en las cadenas de valor que propician la competitividad de las organizaciones.

Conviene señalar que las organizaciones actúan hoy en un mercado global y dinámico, donde sobrevivirán únicamente las que tengan la mayor agilidad para identificar y aprovechar las oportunidades. Por tal razón, dentro de las estrategias de mercadeo de las empresas uno de los factores críticos de éxito consiste en penetrar en nuevos mercados, contar con tecnología de punta ágil y flexible, con el fin de responder a sus requerimientos, personal capacitado para sacar el máximo provecho y adelantar procesos de gestión de conocimiento que orienten a la organización hacia el alcance de los objetivos institucionales.

Según Bustos y Moreno (2003), hasta hace pocos años las pequeñas y medianas empresas (PYMES) aún podían competir sin tecnología informática y de comunicación. Hoy en día competir sin estos dos elementos básicos es imposible, y las empresas que se resistan a actualizarse están prácticamente condenadas al fracaso, debido al bajo nivel de desarrollo tecnológico interno en el área de la producción, la falta de herramientas y aplicaciones informáticas y telemáticas para ejecutar sus actividades administrativas, y no contar con un programa de capacitación permanente a todo el personal de la organización, tanto a nivel operativo, táctico como estratégico de las pequeñas y medianas empresas (PYMES), quizás, uno de los principales factores que más inciden en el bajo nivel competitivo de las organizaciones.

Finalmente, la presente investigación se propone describir el nivel de conocimiento de las TIC por parte de las PYMES exportadoras de Bucaramanga, con el propósito de determinar sus necesidades de capacitación del personal y de infraestructura tecnológica, y dar a conocer a las PYMES dicho análisis, y así contribuir a resaltar la importancia del conocimiento y uso de las TIC, como apoyo para que sean competitivas y aprovechen las oportunidades del mercado internacional.

Las reflexiones planteadas llevaron a los autores de este trabajo a formular una pregunta que orienta su investigación: ¿Cuáles son el conocimiento y el uso de las TIC que tienen los empresarios de las PYMES exportadoras de la ciudad de Bucaramanga? Esta pregunta permitió la organización del estudio y el desarrollo del trabajo de campo de los investigadores.

\section{Métodos}

El diseño de la investigación desarrollada es de tipo no experimental; corresponde a un estudio transversal descriptivo, donde se analizan las variables objeto de dicho estudio. Como se enunció desde el inicio, la población de interés son las PYMES exportadoras de Bucaramanga; se revisaron los datos suministrados por la Cámara de Comercio correspondientes a 73 de ellas; por ende, la población total corresponde a la muestra, teniendo como base que la población es pequeña (en este caso, 73 PYMES exportadoras en Bucaramanga).

Por lo anterior, se debe considerar la totalidad de la población, para que sea representativo respecto en cuanto al tamaño de la muestra. Cabe mencionar que solo 61 de las entidades elegidas colaboraron con el desarrollo de la encuesta y el suministro de la información. Este tipo de muestro es opinático, corresponde a la categoría de muestreo por cupos $^{5}$, pues la característica común de los elementos de la muestra es que se trata de PYMES exportadoras de Bucaramanga.

El instrumento de recolección de información primaria elegido es la encuesta, que se origina y se diseña a partir de la necesidad de obtener la información pertinente para el análisis de las variables por considerar y la posterior validación de la hipótesis.

Las encuestas se aplican a cada uno de los gerentes o propietarios y administradores, así como a los empleados responsables de los procesos tecnológicos, a través de preguntas cerradas de selección múltiple, con múltiple respuesta y única respuesta, donde se establecen categorías relacionadas con el conocimiento de las TIC, las estructura tecnológica y los tipos de capacitación que recibe el personal; es decir, preguntas que faciliten su análisis y el procesamiento de la información, y orientadas de acuerdo con los objetivos y los propósitos planteados para la investigación.

Fuentes secundarias: registros existentes en las diferentes entidades regionales y nacionales, y entre las cuales se incluyen: archivos de entidades como la Cámara 
de Comercio de Bucaramanga, el Servicio Nacional de Aprendizaje (SENA), seccional Bucaramanga, la Asociación Colombiana de Pequeños Industriales (ACOPI), seccional Santander, Proexport Colombia, y el Ministerio de Comercio y Turismo.

Finalmente, se analizaron las características que debe tener una PYMES exportadora para el desarrollo apropiado de sus actividades, según las condiciones de la apropiación de las TIC en sus procesos productivos, establecidos, a su vez, según la clasificación sugerida por un estudio desarrollado por el Programa de las Naciones Unidas para el Desarrollo (PNUD) y Asia-Pacific Economic Cooperation (APEC 2007).

\section{Resultados}

De acuerdo con las cifras del DANE (2005), en Colombia las microempresas conforman el $96,4 \%$ de los establecimientos; las pequeñas empresas, el 3,0\%; las medianas, el $0,5 \%$, y las grandes, el $0,1 \%$. Por personal ocupado, las microempresas representan el 50,3\% del empleo; las pequeñas, el $17,6 \%$; las medianas, el $12,9 \%$, y las grandes, el $19,2 \%$. El estudio realizado por los investigadores arrojó también que el $85,2 \%$ de las PYMES de Bucaramanga desempeñan actividades económicas relacionadas con la transformación, el $8,2 \%$ corresponde a los servicios y el $6,6 \%$ se dedica a la comercialización (Tabla 1). Las PYMES de Bucaramanga representando la mayor parte de la actividad productiva de la región como es el caso de las confecciones y el calzado.

Tabla 1. Clasificación de las PYMES encuestadas

\begin{tabular}{|l|r|r|}
\hline \multicolumn{1}{|c|}{ Clasificación PYMES } & PYMES & \multicolumn{1}{c|}{ Porcentaje } \\
\hline Transformación & 52 & 85,2 \\
\hline Servicio & 5 & 8,2 \\
\hline Comercialización & 4 & 6,6 \\
\hline Total & 61 & 100,00 \\
\hline
\end{tabular}

Fuente: investigación de los autores.

En lo relacionado con la percepción que tienen de las TIC los empresarios, es importante considerar el estudio realizado en Octubre de 2008 por la Red Global de Exportación (RGX), en conjunto con DHL Express y SAP, presentó los resultados del primer estudio regional "Internet y las nuevas tecnologías como herramientas para Las Pequeñas y Medianas Empresas (PYMES) exportadoras.' La información permitió identificar estrategias que incrementen en forma exitosa los negocios. Del estudio realizado con una muestra de 691 PYMES latinoamericanas, pertenecientes a distintas ramas de actividad se extrajeron varios ejes importantes: algunas empresas exportadoras acceden a Internet por diferentes dispositivos, más allá de la clásica PC y que la mayoría, percibe que este medio impactó en la productividad de sus negocios, así como en la reducción de $\operatorname{costos}^{8}$.
De acuerdo con lo obtenido a partir de la recolección de la información para el desarrollo de la investigación, los empresarios están muy de acuerdo en que las TIC son fundamentales para el crecimiento de la empresa (Tabla 2); además, las consideran importantes para apoyar los procesos de exportación, pues reconocen que tienen dificultades cuando no cuentan con las tecnologías apropiadas para mantenerse competitivos. Cabe destacar cómo hay PYMES que consideran fundamental contar con las TIC, sin importar el tamaño o la actividad económica de la empresa, y que son necesarias para desarrollar los procesos de exportación que les permitan cumplir con las exigencias de sus clientes. 
Tabla 2. Percepción de los empresarios sobre si las tecnologías de la información y la comunicación (TIC) contribuyen al crecimiento de su empresa

\begin{tabular}{|l|r|r|r|r|}
\hline \multicolumn{1}{|c|}{ Afirmación } & Muy de acuerdo & \multicolumn{1}{|c|}{ De acuerdo } & En desacuerdo & \multicolumn{1}{c|}{ Total } \\
\hline $\begin{array}{l}\text { TIC contribuyen al } \\
\text { crecimiento de la } \\
\text { empresa }\end{array}$ & 49 & 11 & 1 & 61 \\
\begin{tabular}{l} 
Porcentaje (\%) \\
\hline
\end{tabular} & 18 & 1,6 & 100 \\
\hline
\end{tabular}

Fuente: investigación de los autores.

Por otra parte, en el estudio realizado por SAP Venezuela, Roberto Woord (2008), Director de Riesgo País, para América Latina, del Economist Intelligence Unit, asegura que entre los problemas clave propios de las PYMES se incluye el hecho de que estas enfrentan una oferta limitada de profesionales calificados y de alto potencial. Se les pidió a los encuestados que describieran el mercado laboral actual para profesionales calificados y de alto potencial en América Latina. Casi $66 \%$ de los ejecutivos se quejaron de una escasez de talentos; el $11 \%$, de una limitada oferta de talento, y el $52 \%$, de que las habilidades de los profesionales no son acordes con las necesidades de las PYMES, y de que sostienen una competencia por talento con las grandes empresas ${ }^{9}$.

En el análisis realizado en esta investigación al talento humano se deja ver como muy preocupante el nivel de capacitación que tienen los funcionarios en el manejo de TIC: solo el 34,4\% de ellos ha recibido formación (Tabla 3). Este porcentaje es bajo, porque, según eso, no se ha recibido entrenamiento o no se ha recibido formación en TIC; ello, quizá, originado por fallas en la difusión de los eventos por parte de las entidades estatales que deben impulsar el conocimiento de las tecnologías en el país, esta deficiencia representa un representa un $65,6 \%$. (Tabla 4).

Como el talento humano es clave en el funcionamiento de las PYMES exportadoras de Bucaramanga, las entidades estatales que impulsan el conocimiento de las tecnologías en el país deben diseñar estrategias de capacitación que motiven a los empleados y a los empresarios en la utilización de las TIC.

Tabla 3. Capacitación en TIC del personal

\begin{tabular}{|l|r|r|}
\hline Capacitación en TIC del personal & Respuestas & Porcentaje (\%) \\
\hline Sí & 21 & 34,4 \\
\hline No & 40 & 65,6 \\
\hline Total & 61 & 100,0 \\
\hline
\end{tabular}

Fuente: investigación de los autores.

Tabla 4. Motivos para no asistir a la capacitación

\begin{tabular}{|l|r|r|}
\hline \multicolumn{1}{|c|}{ Motivos para no asistir a la capacitación } & Respuestas & Porcentaje (\%) \\
\hline Horarios no adecuados & 20 & 32,8 \\
\hline No hay difusión del evento & 40 & 65,6 \\
\hline Calidad de la capacitación & 1 & 1,6 \\
\hline Total & 61 & 100,0 \\
\hline
\end{tabular}

Fuente: investigación de los autores.

Continuando con el análisis del uso de las tecnologías de la información y la comunicación en la PYMES, es muy importante considerar el estudio presentado en junio 2009 por la empresa Red Global de Exportación (RGX), que presentó los resultados de la investigación titulada "La innovación como motor para el desarrollo competitivo exportador de las PYMES", realizada en enero, con el patrocinio de Avaya. Su objetivo era cono- cer cuáles son las condiciones, las capacidades y los comportamientos actuales de las PYMES en la inclusión de herramientas TIC, y cómo mejoran el desempeño de su negocio en el comercio exterior. La muestra estuvo conformada por 300 empresas de Colombia, Chile y Argentina (100 por país), de entre 25 y 400 empleados con facturación mínima de US $\$ 45000$, y máxima de US $\$ 1000000$ de dólares, en un período de 6 meses. EI 
resultado del estudio fue la gran aceptación de incluir en las empresas las TIC como motor para generar iniciativas que mejoren los productos o los procesos dentro de las organizaciones ${ }^{10}$.

Las PYMES utilizan los servicios de telecomunicación y transmisión de datos: con los clientes, la utilizada en mayor medida es el correo electrónico, con el $73,8 \%$ de los casos; el chat, con el $41 \%$; las páginas web, con el $24,6 \%$, y la voz sobre Internet, con el $18 \%$. Con los proveedores ocurre algo similar en cuanto al uso del correo electrónico, con el 67,2\%; el chat, con el 16,4\%; la voz sobre Internet, con el 9,8\%, y las páginas web, con el $8,2 \%$. Entre las entidades gubernamentales a las páginas web les corresponde el $62,3 \%$. Con los asesores se usa también el correo electrónico, con el 62,3\%; la voz sobre Internet, con el $18 \%$; las páginas web, con el $8,2 \%$, y el videoconferencia, con el $4,9 \%$.

Por otro lado, en lo relativo a inversiones en TIC, un estudio realizado por la Asociación Nacional de Instituciones Financieras (ANIF) y la empresa de telecomunicaciones UNE, de Medellín, evidenció la falta de inversión que realizan las PYMES en la obtención de tecnologías, pues los empresarios las ven como costosas para las inclusiones de sus procesos. En Colombia tan solo el
$2 \%$ de las PYMES consideran indispensable invertir en tecnología. Tal situación no es exclusiva de las empresas de menor tamaño; mientras que en países avanzados la participación de inversión en TIC dentro del total de inversión de capital es del $4 \%$, en el universo empresarial colombiano dicha participación es de solo el $1 \%$. De tal participación, el $30 \%$ es atribuible a las PYMES, las cuales, en su mayoría, han invertido en TIC ya sea para adquirir o actualizar nuevos equipos, tecnologías o programas. ${ }^{11}$

Finalmente, en lo relacionado con la compra de TIC las empresas analizadas no cuentan con un presupuesto anual establecido para la adquisición, la renovación y la actualización de sus equipos, y tan solo un 31,1\% destina una cifra de entre 4 y 5 millones de pesos como una medida de prevención; principalmente, porque tiene que ver con equipo de cómputo (Tabla5). Ello exige trabajar conjuntamente con los gremios, el sector de las telecomunicaciones y el Estado Colombiano en propiciar espacios de difusión sobre la importancia de contar con las tecnologías necesarias para enfrentar los requerimientos de los clientes y planes de financiamiento para la adquisición de equipos adecuados a las necesidades de los empresarios.

Tabla 5. Presupuesto anual para la compra de TIC

\begin{tabular}{|r|r|r|}
\hline Presupuesto anual para la compra de TIC & Respuestas & Porcentaje (\%) \\
\hline$\$ 4000000-\$ 5000000$ & 19 & 31,1 \\
\hline$\$ 5000000-\$ 6000000$ & 8 & 13,1 \\
\hline$\$ 10000000-\$ 11000000$ & 5 & 8,2 \\
\hline Más de $\$ 11000000$ & 3 & 4,9 \\
\hline No hay presupuesto & 26 & 42,6 \\
\hline Total & 61 & 100,0 \\
\hline
\end{tabular}

Fuente: investigación de los autores.

\section{Discusión}

Cabe destacar que la mayoría de las empresas consideradas en el presente estudio pertenecen al sector de la transformación, y que contribuyen al crecimiento regional mediante las actividades económicas, como la fabricación de prendas de vestir muy reconocidas en el ámbito nacional. Existe, por otra parte, una percepción positiva entre los empresarios sobre la importancia de utilizar las TIC para los procesos de exportación, sin importar el tamaño ni el capital social que tengan en la actualidad.

El intercambio de conocimiento genera valor a la organización. En tal sentido, "el conocimiento en la organización es un conjunto colectivo de saber y saber hacer. Gestionarlo supone administrarlo y rentabilizarlo, lo cual implica planificar y controlar y conlleva adquisición, mantenimiento, medición y puesta en disponibilidad de los recursos. El conocimiento es la fuente de competitividad de las empresas"12.

Entre tanto, se puede decir que el talento humano en las PYMES requiere una política empresarial orientada a lograr una cultura de gestión del conocimiento, en cada uno de sus miembros, sobre la apropiación de las TIC en sus actividades diarias, buscando mejorar sus competencias laborales. Si bien existe una difusión para mejorar el entrenamiento en la utilización de las TIC, la información oportuna a los empresarios, y en muchos casos los horarios programados no facilitan la asistencia permanente de los empleados a los eventos.

El mundo empresarial de hoy es más tecnológico, y en él mantendrá la ventaja quien mejor use el recurso de estas tecnologías aplicadas y alineadas a una estrate- 
gia de negocios. Hoy por hoy se vive en la era de las comunicaciones. Las distancias son cortas y los negocios se hacen en tiempo real a gran velocidad. Cada cual deberá decidir si juega de acuerdo con las reglas del nuevo juego del comercio mundial, o si se queda aletargado y obsoleto. Permanecer competitivo y actualizar la estrategia de crecimiento mediante el uso herramientas ofrecidas por la tecnología de la información debería ser la verdadera estrategia ${ }^{13}$.

Para empezar, se debe reconocer que las PYMES exportadoras de Bucaramanga no orientan sus procesos productivos, de mercadeo ni financiero aprovechando los beneficios de las TIC. Por lo tanto, se puede afirmar que en las empresas analizadas las TIC no están alineadas con la estrategia de negocios, sino que se las utiliza como un medio para intercambiar información y datos. Todas estas deficiencias abren la oportunidad para explorar nuevos usos de las TIC, que les permitan a las PYMES apalancar sus procesos de exportaciones y centrar sus actividades empresariales y desarrollar una formación apropiada para el talento humano, buscando la competitividad de su sector.

Para terminar, existe en las PYMES estudiadas un desconocimiento sobre la importancia de la planificación financiera, así como en los temas de actualización, renovación y adquisición de TIC, para apoyar los procesos productivos, de comercialización y de actividades administrativas; solo se tienen en cuenta valores que están entre 2 y 3 millones de pesos, tan solo para cumplir con pagos relativos a los servicios de Internet y de mantenimiento de los equipos de cómputo.

\section{Conclusiones}

Horner Reich y Benbassat (2000), postulan que, si bien las TIC no proporcionan una ventaja competitiva per se, sí permiten reforzar el potencial de creación de valor de los recursos de la empresa, si las inversiones en TIC se realizan de forma coordinada con la estrategia empresarial. De hecho, los resultados de la investigación empírica sugieren que para crear valor las inversiones en TIC deben, necesariamente, combinarse con recursos complementarios de las empresas (Hitt y Brynjolfsson, 1997; Brynjolfsson y Hitt, 2000) ${ }^{14}$.

Se destaca, que en promedio, el $93,4 \%$ de los empresarios están en desacuerdo en lo relacionado que no necesitan TIC para sus empresas, debido a que consideran fundamental disponer de las TIC, sin importar el tamaño o la actividad económica, y que son necesarias para el desarrollo de los procesos de exportación que les permitan cumplir con las exigencias de sus clientes.

Es importante considerar mejoras en la capacitación del uso de las TIC básicas y avanzadas por parte del talento humano, ya sea en las empresas mismas o por parte de las entidades estatales, de tal manera que se impulse el conocimiento sobre las TIC, y así se aporte al desarrollo de habilidades del capital humano y a la acometividad de las PYMES exportadoras.

En cuanto al uso que las PYMES hacen de los servicios de telecomunicación y transmisión de datos con el clientes se detectó en esta investigación que predomina el uso del correo electrónico, en el $73,8 \%$ de las empresas observadas, y el del chat, con el $41 \%$; con los proveedores el correo electrónico predomina con el $67,2 \%$; con las entidades gubernamentales, en un $62,3 \%$, las páginas web; entre los asesores usan el correo electrónico el $62,3 \%$, pero aún existen deficiencias en la utilización del e-commerce, como una herramienta de negociación por parte de los empresarios; en la mayoría de los casos, por desconocimiento o desconfianza que genera la Internet para suministrar los datos relacionados con los medios de pago de la empresa.

Otro aspecto importante por considerar en las PYMES exportadoras de Bucaramanga es que falta previsión en la reposición de equipos y la gestión de mejores servicios para su empresa, debido a que el $42,6 \%$ de los empresarios no hace presupuesto para la compra, pues consideran que las TIC son inversiones demasiado costosas, lo cual limita la capacidad de respuesta de las organizaciones, la transformación de la cultura organizacional y el intercambio de información entre las diferentes dependencias para lograr un trabajo más articulado, que evite retrocesos innecesarios en el logro de una comunicación asertiva con los clientes.

\section{Referencias bibliográficas}

Bustos, C. A. Nieto M. L A. \& Rojas M. L. (2003) Pymes colombianas y la gestión del conocimiento. Revista Escuela de Administración de Negocios 47, 110. Recuperado de http://journal.ean.edu.co/index.php/Revista/ article/viewFile/208/198.

Bustos, C. A., Nieto M. L A. \& Rojas M. L. (2003). Pymes Colombianas y la gestión del conocimiento. Revista Escuela de Administración de Negocios 47, 112-115. Recuperado de http://journal.ean.edu.co/index.php/Revista/article/viewFile/208/198.

El reto de las Pymes y el desafío del capital humano. (2008). Recuperado de http://www.tecnologiahechapalabra.com/negocios/PYMES/testimonios/articulo. asp? $\mathrm{i}=2733$

Frediani, D. (2008, 28 febrero). ¿Cuál es la importancia de las TICs para las PyMEs exportadoras? Recuperado de http://www.canal-ar.com.ar/noticias/noticiamuestra. asp? $l d=6581$.

García, C. E, Rialp, C. A \& Rialp, C. J. (2008). Tecnologías de la Información y Comunicación (Tic) y Creci- 
miento de la empresa. Recuperado de http://www.unioviedo.es/egarcia/ice.gcr.pdf.

Recuperado de: http://www.estrasol.com.mx/tecnologias-de-informacion-alineadas-a-la-estrategia.php junio 05 de 2011

Jany, J. (2000) Investigación integral de mercados, $2^{\mathrm{a}}$ ed. Bogotá: McGraw-Hill.

Llano, N. N. (2009, 18 de mayo). Política para la Promoción en el acceso y uso de Tic en Micro, Pequeñas y Medianas empresas Colombianas. Recuperado de http://colombiadigital.net/newcd/component/docman/ doc_details/1249-politica-para-la-promocion-en-el-acceso-y-uso-de-tic-en-micropequenas-y-medianas-empresas-colombianas

Pymes en cifras (2008, 29 de agosto). Recuperado de http://www.misionpyme.com/cms/content/view/107/41/

Quirós, M. (2007). Tecnologías de información y comunicación (TIC) para explotar el potencial de crecimiento de las pymes. Bogotá: ANIF.

Recuperado de http://www.ife.gov.ar/articulo/621/9_de_ cada_10_PYMES_exportadoras_creen_que_las_TICs_ ayudan_a_mejorar_su_negocio.aspx febrero 23 de 2011

Recuperado de http://www.profesorenlinea.cl/universalhistoria/U34MRevolucionTecnologica.htmffebrero 24 de 2011

Zaldívar, C. M. (2006). Apuntes para un enfoque adecuado de la gestión del conocimiento en las organizaciones. Recuperado de http://bvs.sld.cu/revistas/aci/ vol14_5_06/aci15506.htm

\section{Notas}

3 Recuperado de http://www.profesorenlinea.cl/universalhistoria/ U34MRevolucionTecnologica.htm

${ }^{4}$ Zaldívar, C. M. (2006). "Apuntes para un enfoque adecuado de la gestión del conocimiento en las organizaciones". Recuperado de http://bvs.sld.cu/revistas/aci/vol14_5_06/aci15506.htm
${ }^{5}$ JANY, J. (2000). Investigación de Mercados. Bogotá. Pág. 98.

${ }^{6}$ Llano, N. N. (2009, 18 de mayo). "Política para la Promoción en el acceso y uso de Tic en Micro, Pequeñas y Medianas empresas Colombianas". Recuperado de: http://colombiadigital.net/newcd/ component/docman/doc_details/1249-politica-para-la-promocionen-el-acceso-y-uso-de-tic-en-micropequenas-y-medianasempresas-colombianas

7 "Pymes en Cifras" (2008, 29 de agosto). Recuperado de http:// www.misionpyme.com/cms/content/view/107/41/

${ }^{8}$ Frediani, D. (2008, 28 febrero). “¿Cuál es la importancia de las TICs para las PyMEs exportadoras?". Canal AR. Recuperado de: http:// www.canal-ar.com.ar/noticias/noticiamuestra.asp?ld=6581

9 "El reto de las PyMEs y el desafío del capital humano" (2008, 25 de julio). Recuperado de: http://www.tecnologiahechapalabra.com/ negocios/PYMES/testimonios/articulo.asp?i=2733

10 Recuperado de: http://www.ife.gov.ar/articulo/621/9_de_ cada_10_PYMES_exportadoras_creen_que_las_TICs_ayudan_a_ mejorar_su_negocio.aspx febrero 23 de 2011

11 Quirós, M. (2007). Tecnologías de Información y comunicación (TIC) para explotar el potencial de crecimiento de las PYMES. ANIF. ISSN 1909-6100. Bogotá, D. C., pp. 1-2.

12 Bustos, C. A., Nieto, M. L., y Rojas, M. L. (2003). "Pymes Colombianas y la gestión del conocimiento". En: Revista Escuela de Administración de Negocios. Número 47, pp. 112-115. Recuperado de: http://journal.ean.edu.co/index.php/Revista/article/ viewFile/208/198

13 "Tecnologías de información alineadas a la estrategia". Recuperado de:http://www.estrasol.com.mx/tecnologias-de-informacionalineadas-a-la-estrategia.php

14 García, C. E., Rialp, C. A., y Rialp, C. J. (2008, octubre). "Tecnologías de la Información y Comunicación (Tic) y Crecimiento de la empresa". Recuperado de: http://www.unioviedo.es/egarcia/ ice.gcr.pdf 